\title{
Transposition
}

Musique et Sciences Sociales

9| 2021

Musique et sexualité

\section{Musique et sexualité}

\section{Esteban Buch et Violeta Nigro Giunta}

\section{(2) OpenEdition}

Journals

Édition électronique

URL : http://journals.openedition.org/transposition/6251

DOI : 10.4000/transposition.6251

ISSN : 2110-6134

\section{Éditeur}

CRAL - Centre de recherche sur les arts et le langage

\section{Référence électronique}

Esteban Buch et Violeta Nigro Giunta, " Musique et sexualité », Transposition [En ligne], 9 | 2021, mis en ligne le 30 mars 2021, consulté le 23 avril 2021. URL : http://journals.openedition.org/transposition/ 6251 ; DOI : https://doi.org/10.4000/transposition.6251

Ce document a été généré automatiquement le 23 avril 2021

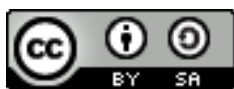

La revue Transposition est mise à disposition selon les termes de la Licence Creative Commons Attribution - Partage dans les Mêmes Conditions 4.0 International. 


\title{
Musique et sexualité
}

\author{
Esteban Buch et Violeta Nigro Giunta
}

1 En août 2020, entre deux vagues de la pandémie de Covid-19, la soprano Chloé Briot dénonce dans La lettre du musicien avoir été victime d'agressions sexuelles de la part d'un chanteur, son partenaire dans l'opéra L'Inondation de Francesco Filidei, mis en scène par Joël Pommerat ${ }^{1}$. Les jours suivants l'affaire prend de l'ampleur dans les médias, surtout lorsqu'à l'annonce du dépôt de plainte de la chanteuse, qui en fait, a eu lieu cinq mois plus tôt, suivent les réactions des maisons d'opéra concernées et la saisie $\mathrm{du}$ parquet par le gouvernement ${ }^{2}$. En septembre, La lettre du musicien publie les résultats d'une enquête menée dans le milieu de l'opéra français par le collectif ComposHer, selon lesquels 83 pour cent des femmes et 39 pour cent des hommes répondants affirment avoir "subi des remarques sexistes dans le cadre de leur métier et leurs études musicales ", 61 pour cent déclarent "avoir déjà vécu une situation de drague insistante, intrusive ou gênante durant leur travail », et 17 pour cent font état d'« un abus de la part d'un ou d'une collègue lors d'une situation de mise en scène $»^{3}$.

2 «Le temps est-il venu du \#MeToo de l'opéra dans l'Hexagone?», se demande la chanteuse et journaliste Juliette de Banes Gardonne en ouverture de ce dernier article. L'année précédente, des dénonciations similaires ont notamment agité le cinéma français. Cependant l'effondrement de la vie musicale à l'automne 2020 suite à la reprise de la pandémie, et l'angoisse de l'ensemble des musiciens face à la question de leur survie professionnelle, rendront provisoirement inaudible ce genre de prise de parole. En janvier 2021, le ministère de la Culture annonce toutefois un programme de lutte contre le harcèlement sexuel dans le domaine de la musique, en évoquant le cas de Chloé Briot ${ }^{4}$. On ne sait ce qu'il adviendra de ces questions lorsque la vie artistique, et la vie tout court, reprendront un semblant de normalité.

3 Or pendant l'affaire de L'Inondation on a fait peu de cas de ce que les épisodes de harcèlement supposés, qui selon la soprano incluaient des comportements déplacés pendant les répétitions et lors des représentations, concernaient un opéra dont le livret prescrit deux scènes sexuelles explicites, tout au moins selon les conventions du genre. L'opéra de Filidei et Pommerat, basé sur une nouvelle publiée en 1929 par l'écrivain soviétique Evgueni Zamiatine, met en scène la crise du couple que forme La femme, 
appelée Sophie et incarnée par Chloé Briot, et L'homme, un personnage sans nom joué par son agresseur supposé. Entre les deux scènes de sexe, leur vie a été bouleversée par La jeune fille, une orpheline de quatorze ans qu'ils ont accueillie chez eux, et qui après une liaison avec L'homme disparaîtra de façon mystérieuse. La première de ces scènes a lieu à l'initiative de La femme, en silence, sur une musique qu'obsède, en figures ascendantes, le désir d'enfant de son mari. Lors de la deuxième, L'homme demande, sur une mélodie statique : "Tu es là? ». « Oui, je suis là », répond-elle par trois fois depuis leur lit, alors qu'il s'approche de son corps sur un crescendo de l'orchestre. Comme on peut le voir sur la captation vidéo du spectacle, la scène culmine cette fois dans le noir d'une ellipse.

4 Il faut dire que cet opéra, dont le livret suit de près le texte de Zamiatine, aborde les violences de genre en tournant le dos aux préoccupations actuelles. La jeune fille, dont l'existence oscille entre la réalité et le fantasme, et qui aujourd'hui pourrait être décrite comme la victime d'une forme d'inceste, est présentée sur scène comme une manipulatrice aussi insouciante qu'aguichante. La femme, une femme au foyer que la jalousie pousse au meurtre de sa rivale, est un être psychologiquement fragile, qui vers la fin, tel un fleuve qui déborde, accouche d'une petite fille en même temps que de ses propres aveux. L'homme, un brave ouvrier entraîné dans l'adultère par sa sollicitude pour l'orpheline, reste jusqu'au bout aux côtés de sa femme malade et enceinte, inquiet devant sa souffrance mais sans assumer la moindre responsabilité. Pendant ce temps, une inondation sans cesse évoquée et jamais perçue se fait la métaphore du monde moral et psychologique des personnages. Au-delà des aspects novateurs de sa dramaturgie et de sa musique, qui diffractent l'action centrale dans celle d'autres personnages habitant le même immeuble, L'Inondation ressemble à une illustration contemporaine de la «défaite des femmes » typique de l'opéra traditionnel, et jadis critiquée par Catherine Clément ${ }^{5}$.

5 Ainsi pourrait-on dire que toute l'affaire se joue autour des didascalies et des indications verbales $d u$ metteur en scène, la soprano insistant sur le fait que, précisément, son partenaire avait outrepassé les contacts prescrits par celui-ci, basculant dans des attouchements non consentis. Cela n'est pas sans rappeler les débats sur les intimacy coordinators au cinéma, qui aux États-Unis ont gagné de l'importance dans l'ère post-Weinstein. Bien sûr, les comportements qu'elle décrit seraient tout aussi inexcusables dans une œuvre sans scènes de sexe; en sens inverse, il ne devrait pas y avoir de limites a priori à ce que l'on peut représenter sur une scène d'opéra. Cependant, dans ce cas précis l'abus dénoncé par la chanteuse dans la vie réelle semble faire écho à la représentation des rapports de force dans le monde de la fiction - à ceci près que, contrairement à La femme, qui s'auto-incrimine dans un moment de folie dostoïevskienne, Chloé Briot a choisi de dénoncer la situation à la justice, inscrivant ainsi d'une manière inattendue l'opéra de Filidei et Pommerat dans l'histoire politique des rapports entre musique et sexualité.

\section{Une impossible cartographie}

6 L'affaire de L'Inondation montre de façon spectaculaire - c'est le cas de le dire - qu'audelà des rapports de pouvoir entre les genres, la sexualité dans les œuvres musicales est imbriquée avec la sexualité dans l'environnement social où elles sont conçues, produites et reçues. S'agissant d'un auteur soviétique, l'association est inévitable avec 
la censure de Lady Macbeth de Chostakovitch par Staline en 1936, qui visait explicitement les moyens sonores utilisés par le compositeur pour représenter la sexualité des personnages. Par la même occasion, l'État totalitaire entendait monopoliser la gestion des rapports de genre et leur représentation sur les scènes de spectacle. En démocratie aussi, genre et sexualité se nouent dans des configurations de pouvoir que ne cessent de transformer l'histoire sociale et politique, ainsi que celle des arts et la littérature, rendant instables les distinctions strictes entre ce qui relève de l'un ou de l'autre. Dans le cas d'œuvres du passé, toute performance associe sur un mode herméneutique les morales sexuelles des deux moments historiques concernés, celui de la création de l'œuvre et celui de la performance elle-même. En témoignent les approches féministes récentes de Lady Macbeth, où le personnage de Katerina, qui selon le livret cède aux avances de Sergueï lors de ce qui constitue de fait une scène de viol, devient une femme active qui mue par son seul désir prend l'initiative de l'acte sexuel à ses risques et périls - la suite de l'opéra mettant en scène une série de meurtres qui culmine avec le suicide de la protagoniste.

7 En fait, les représentations de la sexualité traversent toute l'histoire de l'opéra depuis Monteverdi, y compris bien sûr les piliers du répertoire que sont Mozart et Wagner, ainsi que des figures masculines du vingtième siècle telles que Schreker, Berg, Britten ou Maderna. Plus généralement, on peut considérer que toute musique vocale - y compris dans le vaste univers des musiques populaires chantées - existe en vertu de codes genrés, qu'ils suivent la norme hétérosexuelle ou un modèle gay, lesbien ou queer, et que ces codes définissent avec chaque voix une persona douée d'attraits sensuels tout à fait réels. Par exemple, la sexualisation des chanteuses de jazz est un ingrédient incontournable de leur métier 6 . Aussi, de Madonna à Lady Gaga, d'Elvis à Kaaris, de Bowie et Prince à Björk et Stromae, ou encore Cardi B et Lana del Rey ${ }^{7}$, aucune analyse sérieuse de leur musique ne peut ignorer la performance singulière de leurs êtres sexués, pas plus qu'elle ne saurait, bien sûr, les réduire au statut de sex symbols.

8 Cependant déjà le Kâma Sutra donne une description précise du rôle des instruments de musique dans la chambre où ont lieu les ébats sexuels. La République de Platon ébauche une théorie de la musique d'État qui est aussi un programme d'organisation de la sexualité au moyen de sons organisés. À Rome, certaines fresques de Pompéi montrent des couples d'amants formant de véritables triangles sexuels avec un musicien ou une musicienne. Même l'Église reconnaît les attraits sensuels du son, quitte à tenter de les mettre au service d'une expérience plus " élevée ", ou de ce qu'on a l'habitude - contestable d'ailleurs - d'appeler en termes freudiens la "sublimation ». Saint Augustin se méfiait de ces chanteurs qui, disait-il, prenaient plus de plaisir au fait de chanter qu'à l'amour du Dieu dont ils chantaient les louanges. Au-delà des corps réels qui chantent et de leurs projections fantasmatiques, au-delà des textes et de leur signification rituelle, ce sont la temporalité et le corps ou « grain $^{8}$ » des formes sonores elles-mêmes qui sont ouvertes aux prises somatiques et aux plaisirs érotiques.

9 Or ces éléments formels sont également saillants dans les musiques instrumentales, là où le souffle singulier d'un artiste rencontre les attentes de jouissance des auditeurs. Le côté sexy du saxophone est un lieu commun que les industries culturelles exploitent depuis le début du vingtième siècle, y compris dans les films érotiques et pornos, et qui toutefois n'aurait pas atteint cette prégnance sans les figures temporelles du désir sculptées par Charlie Parker ou John Coltrane. Et, bien entendu, ce n'est pas seulement par le souffle que l'attrait sensuel des musiques vocales rejoint celui des musiques 
instrumentales ; il suffit d'entendre et de voir Martha Argerich ou Yuja Wang au piano pour s'en convaincre. La capacité d'une musique à érotiser ses auditeurs est indépendante de l'anthropomorphisme des instruments ou des sources électroniques concernées, brassées de nos jours par une myriade de ressources informatiques qui, souvent, rendent poreuses les distinctions entre l'organisme et la machine, entre les sons humains et non-humains, entre les sons musicaux et ceux que la coutume ou les goûts tiennent à distinguer de la musique.

10 Cependant, les courbes d'intensité qui animent les différents paramètres sonores de la musique sont des affordances qui, selon les contextes et les dispositions individuelles, peuvent entrer - ou non - en résonance avec les rythmes des plaisirs intimes des individus. C'est ce que savent bien toutes les personnes qui plus ou moins consciemment, et de manières variables selon l'occasion, vivent la danse comme une expérience érotique, qu'il s'agisse des danses de couple, où les individus sont reliés par ce que le tango nomme un abrazo, ou des dancefloors, où chacun évolue de façon autonome en interaction avec ses voisins, dans une sorte de performance sensuelle collective. C'est aussi ce que pratiquent au quotidien les nombreuses personnes qui font de la musique une atmosphère, voire une présence, au sein de leurs vies sexuelles partagées ou solitaires.

11 Ainsi la musique peut-elle s'intégrer aux scripts sexuels dont les êtres humains disposent pour faire vivre leur désir et celui de leurs partenaires ${ }^{9}$. Et cela ne saurait être expliqué seulement en associant un type de musique à un mood, comme le suggèrent les plateformes de streaming avec leurs playlists "soirée romantique » et autres «spéciale Saint Valentin ». Il est tout à fait possible d'entendre "Love to love you baby " de Donna Summer sans ressentir la moindre excitation, tout comme il est possible d'être excité par une œuvre de Bach. Il est vrai, cependant, que le premier morceau, avec les vocalisations orgasmiques de la chanteuse et l'allure disco de son rythme, donne au sexe des prises plus évidentes que le second. En termes techniques, selon l'écologie de la perception, cela correspond à une densité plus élevée des affordances qui connectent les deux domaines de l'expérience humaine.

\section{Sexualités latentes?}

Dresser l'inventaire même le plus sommaire de tous les genres musicaux et toutes les situations où la musique rencontre la sexualité est une tâche vouée à l'échec. À ce propos on peut toujours faire valoir la leçon classique de Michel Foucault, lorsqu'il s'inscrit en faux contre "l'hypothèse répressive ", c'est-à-dire contre l'idée que la culture patriarcale aurait systématiquement réduit au silence tout discours sur la sexualité ${ }^{10}$. Au contraire, la musique n'a jamais cessé de dire le sexe, d'en faire entendre les logiques du désir et les courbes temporelles, de stimuler les fantasmes et d'interpeller les corps. Vu la présence constante de la sexualité dans l'histoire de la musique, qu'elle soit "occidentale » ou pas, on pourrait même s'étonner de ce qu'une revue telle que Transposition doive encore présenter un numéro spécial sur « Musiques et sexualités ", comme s'il s'agissait d'une thématique nouvelle.

13 Et pourtant les études scientifiques à l'intersection de la musique et de la sexualité restent rares d'un point de vue quantitatif, et lacunaires d'un point de vue thématique. Ce dossier vise justement à remédier quelque peu à ces silences du savoir, et à rouvrir une conversation qui, en fait, touche au cœur des pouvoirs de la musique. Cependant, le 
contraste entre une musique qui n'aurait cessé de faire entendre les sons du sexe, et des études musicales qui n'auraient cessé d'éviter de s'en faire l'écho, exige une clarification des termes du débat. Le livre Feminine Endings de Susan McClary doit son titre anglais au vocabulaire utilisé par les théoriciens du XIX ${ }^{e}$ siècle pour parler de phénomènes musicaux qui n'avaient pas de lien direct ni avec le sexe, ni avec le genre, mais qui mobilisaient des lieux communs genrés et sexistes, tels que l'association naturalisée entre la féminité et la faiblesse ${ }^{11}$. Richard Wagner, qui faisait de la sexualité un thème récurrent de ses drames musicaux sur un mode souvent transgressif, déployait également un imaginaire genré dans ses textes théoriques, par exemple en décrivant le drame musical comme une union entre la poésie et la musique, présentées comme respectivement masculine et féminine ${ }^{12}$. Aussi, tous les discours sur l'« art dégénéré », depuis Max Nordau jusqu'aux persécutions nazies, reposent sur la reconnaissance des effets sexuels de l'art moderne, y compris la musique, sur les corps et les subjectivités ${ }^{13}$.

La sexualité et le genre, ainsi que leur nouage intime dans le dispositif de l'hétéronormativité ${ }^{14}$ sont donc bel et bien présents jusque dans les discours réactionnaires. L'inscription des théories de la musique dans la trame culturelle des discours sur la sexualité ou, si l'on veut rester foucaldiens, dans celle de la sexualité en tant que discours, est un chantier théorique et empirique encore largement en friche. C'est pourquoi nous proposons dans ce dossier de renouer de manière critique avec certains textes marquants des années 1990, qui ont constitué à cet égard un moment charnière, et dont l'influence s'étend jusqu'à nous par des chemins plus ou moins directs. En amont de cette époque, cependant, c'est probablement à Theodor W. Adorno que l'on doit la première formulation d'une " théorie sexuelle » de la musique, dans des termes à bien des égards proches de l'horizon théorique contemporain. Dans son essai Über jazz, écrit en 1936 en Angleterre peu avant son départ pour les États-Unis, Adorno écrit que le rythme du jazz est le rythme même de l'acte sexuel, avant d'en tirer les conséquences pour sa théorie de l'industrie culturelle ${ }^{15}$. Prenant à rebours l'hypothèse freudienne d'un contenu sexuel latent dans les rêves ou les névroses, que l'analyse devrait rendre manifeste pour le sujet, il affirme que le jazz se caractérise par un contenu sexuel manifeste dont le contenu latent n'est autre que la domination sociale, traduite dans l'aliénation des individus par rapport à leur désir - ou, en termes freudiens, dans une forme de castration. Selon lui, le fait que des musiciens noirs soient le plus souvent à l'origine des plaisirs du jazz n'est en rien dû à leurs origines raciales, mais bien à cette condition de dominés. La contestation fréquente de son diagnostic sur le jazz, sur laquelle il n'est pas possible de revenir $\mathrm{ici}^{16}$, ne fait que confirmer la puissance heuristique de ce discours critique sur les rapports entre musique et sexualité, que curieusement l'auteur aura ensuite mis en sourdine pour le reste de sa $\mathrm{vie}^{17}$.

\section{Le moment de la new musicology}

15 À cette présence latente de la sexualité dans les études musicales traditionnelles succède dans les années 1980 un véritable coming out qui, toutefois, prend d'abord et surtout la forme d'un intérêt pour les questions de genre. Ce mouvement a comme épicentre les États-Unis, suite à l'influence croissante du féminisme et des études de genre, mais une impulsion décisive lui vient de France, imbriquée dans l'impact de la 
French theory sur la pensée théorique en général, et notamment de la déconstruction derridienne ${ }^{18}$. Dans ce contexte, le livre de Catherine Clément de 1979, traduit en 1988, souligne que, bien que les femmes soient souvent au cœur de l'opéra, le genre opératique n'a rien d'une libération féministe: "Tout au contraire : elles souffrent, elles crient, elles meurent [...]. Elles s'exposent, décolletées jusqu'au cœur, luisantes de larmes, au regard de ceux qui viennent jouir de leurs supplices feints. Pas une n'en réchappe, ou si peu ${ }^{19} . .$. ». Laissant de côté la musique pour se concentrer sur les livrets, Clément invite à entendre, derrière les voix adorées, les mots brutaux qui les tuent. Ainsi, pour l'autrice, l'opéra exprime-t-il la " défaite des femmes ", condamnées par le pouvoir patriarcal à renoncer à leurs désirs, à souffrir et à mourir. La beauté et virtuosité de leurs voix ne font qu'enrichir le spectacle macabre qui culmine avec la mise à mort de la soprano pour le plaisir d'un public d'hommes, certes accompagnés de leurs femmes qui, ainsi, assistent à l'achèvement de leurs semblables.

Clément défend l'idée que pour ne pas perpétuer cette violence il faut commencer par montrer qu'elle existe. C'est là une position partagée au sein du courant de la new musicology, la «nouvelle musicologie » qui fait irruption dans le mainstream lors du congrès de l'American Musicological Society de 1990 à Oakland, avec des panels sur l'homosexualité, le féminisme et le rap, et la First Feminist Theory and Music Conference de 1991 à Minneapolis. Dans ce contexte, l'orientation sexuelle des compositeurs fait l'objet de travaux tels que ceux de Maynard Solomon sur Schubert, qu'il décrit comme homosexuel en se basant sur ses écrits, son comportement en société, son refus du mariage, une certaine antipathie pour les femmes, et ses relations proches avec des hommes ${ }^{20}$. Non sans soulever des scepticismes et des résistances, comme le signale Susan McClary en pointant un parallèle avec la censure du photographe Robert Mapplethorpe en 1991, ou les restrictions des droits des homosexuels dans l'armée états-unienne en $1992^{21}$.

Dans ce débat, Mcclary défend l'importance de considérer la sexualité au sens large pour comprendre les contextes de création. Selon elle, les fantaisies sexuelles supposées de Schubert jettent une nouvelle lumière sur la "musique absolue ", voire fondent une nouvelle interprétation de ce répertoire. Lawrence Kramer soutient pour sa part que les formes du soi considérées normales par la culture occidentale promeuvent et rationalisent la violence contre les femmes, et analyse les œuvres musicales du xIX ${ }^{e}$ siècle pour comprendre les évolutions historiques de la conception du désir ${ }^{22}$. En 1994, l'ouvrage Queering the Pitch ${ }^{23}$ met de côté les définitions essentialistes de la sexualité pour insister sur la performativité, convergeant avec les thèses de Judith Butler dans Gender Trouble, paru en 1990. Suzanne Cusick, qui revendique le lesbianisme comme une échappatoire aux structures du pouvoir patriarcal, y explore les conséquences de son orientation sexuelle sur son métier de musicologue et de musicienne, en décrivant une interprétation à l'orgue des Variations canoniques de Bach comme un acte sexuel ayant pour partenaire la musique elle-même : "What if music IS sex? ", se demande-t-elle ${ }^{24}$. En 1996, John Corbett et Terri Kapsalis écrivent un article remarqué sur l'orgasme féminin dans les musiques populaires, ouvrant ainsi la porte à une intégration de la musique dans un domaine plus vaste, qui ne s'appelle pas encore les sound studies ${ }^{25}$.

Le texte marquant de cette période reste toutefois le livre Feminine Endings de McClary, paru en 1991, dont l'apport le plus saillant est aussi le plus controversé : selon elle, l'organisation du temps musical dans le système tonal reporte la gratification en créant 
chez l'auditeur l'attente d'un retour à la tonique, une délivrance finale qu'elle décrit comme une "éjaculation métaphorique ". Cette approche provoque le scandale dès 1987 lorsqu'elle propose d'entendre, à la suite de la poétesse féministe Adrianne Rich, la réexposition du premier mouvement de la Neuvième symphonie de Beethoven comme "l'un des moments les plus horribles de toute la musique, là où la frustration d'une cadence soigneusement préparée génère une énergie qui finit par exploser dans la rage meurtrière d'un violeur incapable d'atteindre la jouissance ${ }^{26} »$. Dans son livre, McClary retranchera de ce passage la figure du violeur, sans pour autant renier sa critique radicale du lien entre le système tonal et le patriarcat ${ }^{27}$.

Par la même occasion, elle entend décloisonner la musique classique et la musique populaire, rejoignant ainsi un questionnement sur la place de femmes au sein du canon musical qui était celui de Marcia Citron depuis la fin des années $1980^{28}$. Au même moment, la question de la sexualité fait irruption dans les études sur la popular music, avec notamment la section "Rock and Sexuality » d'un livre édité en 1990 par Simon Frith et Andrew Goodwin ${ }^{29}$. En France, Eugène Lledo propose en 1991 une lecture de l'histoire du rock entre 1950 et 1980 suivant les points de contact entre musique et sexualité $^{30}$. Cela reflète un intérêt plus ancien, car déjà en 1978 Frith et Angela McRobbie signalent par exemple la dimension phallique de la guitare électrique, et, au risque d'essentialiser le lien entre les genres et les goûts, comparent la consommation passive et féminine de la pop par les adolescentes, et la consommation active et masculine du cock rock. Aussi, en 1984 Sue Wise s'interroge sur sa fascination érotique pour Elvis Presley, et la dissonance apparente entre ce désir d'adolescente et son militantisme féministe ultérieur, autrement dit entre logique sexuelle et logique de genre. C'est contre la tentation essentialiste que va réagir en 1999 Susan Fast en faisant une enquête ethnographique sur les femmes fans de Led Zeppelin, qui vont décrire les solos de guitare de Jimmy Page comme des « orgasmes électriques prolongés ${ }^{31}$ ».

\section{L'apport des sciences sociales}

Comme son nom l'indique, dans les années 1990 la new musicology émerge comme un courant nouveau au sein de la musicologie, sans pour autant remettre en cause la définition institutionnelle de la discipline. Les études sur la popular music, à leur tour, s'ouvrent aux questions de genre et de sexualité suite à une dynamique intellectuelle qui leur vient surtout de leurs objets, sans remettre en cause leur périmètre habituel. Cependant, à cette époque les sciences humaines et sociales favorisent de plus en plus le dialogue interdisciplinaire, voire assument un certain esprit transdisciplinaire, typique notamment des cultural studies. En sens inverse, les disciplines traditionnelles que sont l'histoire, la sociologie ou l'anthropologie s'intéressent de plus en plus à des objets qui jusque-là étaient le domaine réservé de disciplines spécifiques telles que la musicologie elle-même, les études littéraires, ou encore l'histoire de l'art.

21 C'est de ces mêmes années 1990 que l'on peut dater une approche des rapports entre musique et sexualité résolument ancrée dans les sciences sociales, telle qu'entend la défendre et l'illustrer ce numéro spécial de Transposition. Il en résulte un intérêt pour des questions qui ne sauraient être réglées par une analyse ou une herméneutique des seuls objets musicaux, comme le faisait volontiers la new musicology. Il s'agit plutôt de comprendre comment, concrètement, la musique et la sexualité s'articulent dans des pratiques, comment cette articulation fait sens pour les individus sous la forme de 
représentations et d'expériences, et comment, à leur tour, ces pratiques, ces représentations et ces expériences nourrissent le travail créatif des musiciens et des musiciennes, selon des logiques sociales et politiques qui sont notamment des rapports de genre. C'est pourquoi le présent dossier s'ouvre sur la réédition de deux textes parus dans les années 1990, précédés d'introductions spécialement rédigées par leurs autrices, en guise de retours réflexifs qui sont aussi des coups d'œil informés sur l'évolution du champ.

Le premier de ces textes, celui d'Annegret Fauser, fut rédigé et présenté en français en 1992 lors d'un colloque consacré à Jules Massenet, dont les actes ne parurent qu'en $1999^{32}$. Il concerne "l'élément érotique» dans l'œuvre du compositeur, et singulièrement l'opéra Esclarmonde, créé en 1889 à l'Opéra-Comique. L'autrice, née en Allemagne et aujourd'hui professeure à l'université de Caroline du Nord à Chapel Hill (États-Unis), était alors doctorante, en stage à l'École normale de Paris. Spécialiste de la musique française à la Belle Époque, à laquelle elle dédie sa thèse, elle tâche dans cet article pionnier de cerner ce qu'elle appelle l'« élément érotique » en articulant une analyse musicale non formaliste, proche du new criticism prôné notamment par Joseph Kerman $^{33}$, et une histoire de la réception. Elle recueille des traces aussi éloquentes que les mots de ce critique qui, rendant compte de la création de l'opéra dans la Revue des Deux-Mondes, s'efforce de dire la sexualité sans la dire, quitte à avouer son embarras avec un clin d'œil : « Jamais encore on n'avait, je crois, fait une description sonore aussi fidèle, aussi détaillée, de la manifestation physique des tendresses humaines (vous voyez que je tâche de m'exprimer convenablement) ${ }^{34}$ ».

En se concentrant plus sur la musique que sur les textes, Fauser semble arriver à une conclusion opposée à celle de Clément: dans Esclarmonde de Massenet, l'érotique musicale de l'hérö̈ne - une magicienne aux pouvoirs fabuleux - lui permet de gagner de l'influence, d'arriver à ses fins, de tenter une transgression des structures patriarcales, presque une émancipation. Les moyens musicaux avancés donnent forme et matière au personnage féminin pour exprimer son désir, sur un mode actif alors jugé peu compatible avec son sexe. Cependant, la résolution de l'histoire répond aux idéaux bourgeois de son époque : Esclarmonde, déchue de ses pouvoirs magiques, doit se plier au désir de son père l'Empereur, qui l'a promise au vainqueur d'un tournoi, heureusement gagné par celui qu'elle aime déjà en secret. Ainsi, moyennant la convergence apparente du désir et du devoir, l'ordre patriarcal est rétabli dans un happy end.

L'autre texte réédité dans ce dossier est de la sociologue anglaise Tia DeNora, professeure à l'Université d'Exeter, au Royaume Uni. Intitulé "Music and Erotic Agency ", il date de 1997, et fait partie du chantier de l'autrice sur « la musique dans la vie quotidienne ", qui aboutira à un ouvrage désormais classique ${ }^{35}$. Ce texte est peutêtre le premier à avoir esquissé une étude sociologique de la musique dans la vie sexuelle de personnes réelles, en l'occurrence une population d'étudiantes, dont l'expérience fut recueillie au moyen d'entretiens. Ce faisant, DeNora retrouvait des questions plus générales : quels sont les liens entre nos vies quotidiennes et les produits culturels que nous consommons? Comment l'agentivité érotique peut-elle être, selon ses termes, "musicalement composée" ? Si l'on admet que la musique est une ressource pour discipliner le corps, les émotions et les actions, peut-on écouter de la musique dans le sexe ? Par la même occasion, elle contribuait au tournant féministe des sciences sociales, car les témoignages de ses enquêtées - toutes des femmes - 
convergent dans des anecdotes sur l'incapacité des hommes hétérosexuels à trouver la bonne musique et le bon comportement, autrement dit à tenir jusqu'au bout le script hétéronormatif de la rencontre sexuelle, dans le respect des désirs et des goûts de leurs partenaires.

L'intensité des débats et la qualité des textes produits dans les années 1990 contrastent avec la production moins significative des années qui ont suivi. Entre-temps, l'herméneutique sociale et politique des œuvres musicales est admise, ainsi que l'intégration des questions de genre dans la pratique des musicologues, des sociologues et des historiens de la culture. C'est tout naturellement, pour ainsi dire, qu'un ouvrage de référence de 2003 sur Debussy inclut un texte de Julie McQuinn sur l'érotisme dans sa musique ${ }^{36}$. Sur Madonna seulement la bibliographie accumulée est impressionnante, et inclut bien sûr des réflexions sur la performance de sa sexualité. On peut par ailleurs mentionner les réflexions de Robert Fink de 2005 sur la répétition en musique, ou le volume de 2010 Earogenous Zones, qui fait la part belle à la musique au sein d'une réflexion sur le sonore au cinéma en général ${ }^{37}$.

Parmi d'autres références de la dernière décennie, comptons l'article de Federico Lazzaro sur les Chansons madécasses de Ravel ${ }^{38}$, le livre de Ted Gioia sur les chansons d'amour ${ }^{39}$, l'étude d'Ivan Raykoff sur le cliché du pianiste romantique ${ }^{40}$, ou encore l'enquête du linguiste François Péréa sur «ce qu'on se dit au lit », que l'on peut lire désormais comme un texte de sound studies ${ }^{41}$. Danielle Sofer, pour sa part, déploie un questionnement analytique sur la sexualité dans la musique contemporaine ${ }^{42}$. Enfin, faute d'aborder ici tous les genres musicaux où les questions de sexualité sont prédominantes, on peut mentionner quelques études parues en Amérique Latine dans le sillage de Tango and the Political Economy of Passion de Marta Savigliano ${ }^{43}$ : un numéro spécial de la revue mexicaine Versión paru en 2014, et les ethnographies de María Julia Carozzi et Mercedes Liska sur les milongas de Buenos Aires ${ }^{44}$. Enfin, notons que les pratiques artistiques militantes en Argentine sont au centre de la démarche du groupe Opera Queer, interviewé par les chercheuses Jazmín Tizcornia et Giselle Méndez pour ce numéro.

Ainsi, ces dernières années, la sexualité est en passe de redevenir un objet marquant des études musicales, en liaison avec l'ouverture aux sciences sociales et l'importance prise par les études de genre. La frontière thématique entre genre et sexualité est toutefois poreuse, les études sur la sexualité au sens restreint restant minoritaires face au questionnement des rapports de pouvoir en général, focale de plusieurs groupements de chercheurs. En France, un repère important a été la traduction en 2015 de Feminine Endings de Susan McClary, rebaptisé Ouverture féministe, et précédé d'un avant-propos de Catherine Deutsch ${ }^{45}$. Celle-ci, enseignante à Sorbonne Université, est par ailleurs autrice d'études décisives sur la sexualité dans la musique de la Renaissance ${ }^{46}$ au sein du Cercle de recherche interdisciplinaire sur les musiciennes (CReIM) et du programme Genre, musique et musiciennes (GeMM) à l'Institut de recherche en Musicologie (IReMUS), dont la production sur les pratiques musicales des femmes est par ailleurs conséquente. Dans le même élan, la Philharmonie de Paris a également traduit l'ouvrage de Wayne Koestenbaum de 1993 Anatomie de la folle lyrique, sur les liens entre opéra et homosexualitée ${ }^{47}$.

28 En 2013, le troisième dossier thématique de Transposition, Musique et théorie queer, incluait une étude ethnographique d'Esperanza Miyake sur un chœur lesbien et gay, et un entretien avec Annegret Fauser où celle-ci évoquait son parcours au sein d'un 
champ musicologique élargi, croisant le genre et la sexualité avec l'histoire et la politique $^{48}$. À l'EHESS, le séminaire collectif Atelier d'histoire culturelle de la danse aborde dès 2008 les corps pris dans le mouvement de la musique, témoin les travaux de Sophie Jacotot sur les danses américaines à Paris dans l'entre-deux-guerres, ou ceux d'élizabeth Claire sur la valse ${ }^{49}$. Enfin, depuis 2016, le séminaire Musique et politique animé par Esteban Buch a donné lieu à une étude sur les rapports entre climax musical et orgasme chez Debussy ${ }^{50}$, et à l'organisation de plusieurs journées d'études sur le thème Musique et sexualité.

\section{Présentation du dossier}

Le dossier thématique Musiques et sexualités de ce numéro de Transposition se compose de la réédition des articles de Annegret Fauser et Tia DeNora, et de la publication de cinq études récentes. Le premier de ces articles inédits est celui d'Aurore Flamion, «L'agentivité d'une île: Les Stigmatisés (1918) de Franz Schreker ». L'autrice part de la réception de cet opéra à la suite de sa création à Francfort, en remarquant que les critiques font volontiers référence à la présence de la sexualité dans le livret, la musique et la vie du compositeur. Elle se recentre ensuite sur l'œuvre et les matériaux musicaux eux-mêmes, qui selon elle proposeraient au public de vivre une forme de stase érotique. Son analyse se déroule en deux temps. Elle prend d'abord le début du III acte et la présentation de l'île de l'Élysée construite par le protagoniste Alviano Salvago, en principe pour célébrer la beauté, et qui dans les faits attise la débauche des personnages. En réponse aux interprétations qui font de l'île une projection narcissique du protagoniste, elle suggère que celle-ci possède en soi la capacité de mettre les spectateurs dans une situation érotique. Dans un deuxième temps, elle analyse les textures orchestrales caractéristiques du compositeur: le Klang (son) schrékerien devient un élément dramaturgique à part entière par l'attrait «mystique, sensuel, dangereux et irrépressible » qu'il exerce sur les personnages, avec des timbre-sons complexes qui contribuent à l'induction d'une stase. Elle en conclut que l'effet de l'action de l'île sur les personnages est analogue à celui que le compositeur veut susciter sur son propre public, et où la nature devient un lieu doté de vie.

Dans "L'hyperféminisation des chanteuses japonaises: shôjo kashu et aidoru », Chiharu Chujo et Clara Wartelle-Sakamoto abordent la sexualité des jeunes chanteuses, danseuses et actrices japonaises. En traçant l'évolution historique entre les shôjo kashu ( ( fillettes chanteuses ») des années 1950 et les aidorus («idoles ») contemporaines, leur contribution permet de comprendre un phénomène de sexualisation progressive des corps et du comportement des femmes, dans une logique de marché née des bouleversements politiques et culturels de l'après-guerre. Elles étudient la gestion de l'image de ces femmes et l'adaptation contrainte de leur vie privée en vue d'attirer un public voyeuriste, majoritairement masculin, tout en soulevant indirectement des questions difficiles sur la pédophilie. Selon les autrices, la sexualité, qui reste un sujet réprimé dans la société japonaise, trouve dans ces jeunes chanteuses une voie pour se manifester, dans une logique de fan service.

31 Les liens possibles entre les formes musicales et l'acte sexuel sont au cœur de la contribution de Marion Brachet, "Multiple and Sustained Climaxes in Icelandic PostRock : For an Expansion of the Notion of Musical Climax ». Dans une première partie, l'autrice relève les multiples concordances entre climax musical et climax sexuel, pour 
penser l'orgasme comme une référence formelle pour l'analyse musicale. En partant des modèles d'orgasme classés selon les sexes biologiques, elle incite à dépasser le binarisme masculin-féminin en distinguant trois types de temporalité, à savoir unique, multiple et soutenue. Brachet analyse ensuite des morceaux du post-rock islandais «Popplagio » de Sigur Ròs, « Naros » de Sólstafir et «The Prolonging » de Triptykon comme exemples de climax multiples et soutenus, en opposition à la téléologie des cadences classiques et de la forme sonate, des notions qu'elle contribue ainsi à démasculiniser.

L'article de Toby Young, «La Petite Mort: Techniques of Orgasm in Electronic Dance Music », entreprend une ethnographie des discours sur la sexualité dans l'univers de l'Electronic Dance Music (EDM). À partir d'entretiens réalisés au Royaume-Uni, il montre qu'au contact de cette musique les participants établissent eux-mêmes des parallèles entre leurs expériences de la danse et de la sexualité, notamment par l'utilisation d'un champ lexical commun. Young analyse l'espace sexualisé de la piste de danse comme un safe space où l'on peut rencontrer des partenaires et s'exprimer, et où les expériences sont souvent amplifiées par la consommation de drogues. Prolongeant l'exploration de la notion de climax dans l'article précédent, il observe que dans la musique elle-même la répétition, les progressions de bpm et les résolutions harmoniques s'amplifient jusqu'au moment du «drop». À la place du modèle hétéronormatif de l'orgasme comme point d'arrivée unique, il s'appuie sur les technologies de l'excitation à l'œuvre dans l'EDM pour élargir la discussion aux théories du kink et du BDSM. Cela lui permet d'introduire une notion de jouissance non génitale, essentiellement composée de mécanismes de contrôle et de jeux de pouvoir, au service du plaisir de l'écoute et du mouvement des corps.

Enfin, dans le dernier article, Charlotte Vaillot-Knudsen nous invite à pénétrer les musiques de la Terre, en résonance avec le mouvement de l'écosexe, impulsé dans les années 2000 par les artistes Elizabeth Stephens et Annie Sprinkle. Ces pratiques érotico-artistiques, qui mélangent humour et engagement environnemental, se retrouvent dans les grottes qui sont l'objet de son étude. « De l'orgue au septième ciel. Pour une spéléologie du souffle-désir » vient ainsi clôturer le dossier avec un retour à la question de l'agentivité érotique des environnements sonores, déjà abordée à propos de l'île de Schreker. Dans un univers souterrain sexué et minéral, l'autrice explore en particulier le grand orgue des grottes de Luray en Virginie (États-Unis), tout en se penchant sur l'étymologie du mot " orgue », son histoire et son répertoire, ainsi que sur l'expérience de l'écoute « poético-esthétique du chercheur-spéléologue ».

\section{NOTES}

1. BANES GARDONNE DE Juliette, «La chanteuse Chloé Briot porte plainte pour agression sexuelle », La Lettre du musicien $\mathrm{n}^{\circ}$ 537, en ligne le 19 août 2020.

2. BLAYo Mathilde, "Affaire Briot: les réactions des directeurs d'opéras ", La Lettre du musicien $n^{\circ}$ 537, 20 août 2020 ; "La soprano Chloé Briot accuse un chanteur d'agression sexuelle», Francemusique.fr, 21 août 2020 ; «La soprano française Chloé Briot accuse un chanteur d'agression 
sexuelle ", Lefigaro.fr, 25 août 2020 ; «"Loi du silence à l'opéra" : une soprano porte plainte pour agression sexuelle », L'Obs, 8 septembre 2020 ; «Agression sexuelle présumée contre la soprano Chloé Briot: Bachelot avise le parquet», Libération.fr, 8 septembre 2020 ; "Chloé Briot: une histoire de l'omerta ", ComposHer, 8 septembre 2020, en ligne.

3. BANES GARDONNE DE Juliette, "Sexisme et harcèlement à l'opéra ", La Lettre du musicien $\mathrm{n}^{\circ} 538$ (septembre 2020), p. 13.

4. PIETRALUNGA Cédric et CORDIER Solène, «Roselyne Bachelot veut lutter contre les violences sexuelles dans la musique ", Le Monde.fr, 14 Janvier 2021.

5. CLÉMENT Catherine, L'opéra ou la défaite des femmes, Paris, Grasset, 1979.

6. BUSCATTO Marie, Femmes du Jazz. Musicalités, féminités, marginalisations, Paris, CNRS, 2007.

7. HAMIDI-KIM Bérénice, "Lana Feminism ou les rêves de Lana Del Rey - de "Video Games" à "Chemtrails over the Country Club" ", en ligne : https://aoc.media/critique/2021/02/04/lanafeminism-ou-les-reves-de-lana-del-rey-de-video-games-a-chemtrails-over-the-country-club/

8. BARTHES Roland, «Le grain de la voix ", dans L'obvie et l'obtus. Essais critiques III, Paris, Seuil, 1982.

9. GAGNON John, An Interpretation of Desire: Essays in the Study of Sexuality, Chicago, The University of Chicago Press, 2004.

10. Foucault Michel, Histoire de la sexualité. 1 : La volonté de savoir, Paris, Gallimard, 1976, p. 47.

11. MCCLARY Susan, Feminine Endings: Music, Gender and Sexuality, Minneapolis, University of Minnesota Press, 1991.

12. NATTIEZ Jean-Jacques, Wagner androgyne. Essai sur l'interprétation, Paris, Christian Bourgois, 1990.

13. NORDAU Max, Dégénérescence, Paris, Félix Alcan, 1899.

14. JACKSON Stevi, «Genre, sexualité et hétérosexualité : la complexité (et les limites) de l'hétéronormativité ", Nouvelles Questions Féministes, 2015/2 Vol. 34, p. 64-81.

15. ADORNO Theodor W., "À propos du jazz ", Moments musicaux, trad. et postface Martin Kaltenecker, Genève, Contrechamps, 2003, p. 67-95.

16. DESPLAT-ROGER Joana, Le jazz comme résistance à la philosophie, thèse de doctorat Université Paris-Nanterre, 2020.

17. Buch Esteban, «Sur le jazz d'Adorno comme théorie sexuelle de la culture », dans L'écho du réel, CRIGNON Cyril, LAFORGE Wilfried et NADRIGNY Pauline (eds.), Paris, Éditions Mimésis, 2021, sous presse.

18. KALTENECKER Martin, "L'analyse considérée comme une guerre continuée par d'autres moyens. Remarque sur Heinrich Schenker, Milton Babbitt et la New Musicology », dans BUCH Esteban, DONIN Nicolas et FENEYROU Laurent (eds.), Du politique en analyse musicale, Paris, Vrin, 2013, p. 183-204. CUSSET François, French Theory. Foucault, Derrida, Deleuze et Cie et les mutations de la vie intellectuelle aux États-Unis, Paris, La Découverte, 2003.

19. CLÉMENT, op. cit., p. 24.

20. solomon Maynard, "Franz Schubert's "My Dream" », American Imago 38, p. 137-154, 1981 ; solomon Maynard, "Franz Schubert and the Peacocks of Benvenuto Cellini ", $19^{\text {th }}$ Century Music $\mathrm{XII} / 3,1989$.

21. MCCLARY Susan, "Music and Sexuality: On the Steblin/Solomon Debate ", $19^{\text {th }}$ Century Music XVII/1, 1993, p. 86.

22. KRAMER Lawrence, Music as Cultural Practice. 1800-1900, Berkeley, University of California Press, 1990 ; KRAMER Lawrence, After the Lovedeath: Sexual Violence and the Making of Culture, Berkeley et Los Angeles, University of California Press, 1997.

23. BRETt Philip, thomas Gary, wood Elizabeth (eds.), Queering the Pitch: The New Gay and Lesbian Musicology, Routledge, New York, 1994.

Transposition, 9 | 2021 
24. CUSICK Suzanne G., "On a Lesbian Relationship with Music: A Serious Effort Not to Think Straight ", in Queering the Pitch, op. cit., p. 67-83.

25. CORBETT John et KAPSALIS Terri, «Aural Sex: The Female Orgasm in Popular Sound », The Drama Review 40/3 (automne 1996), p. 102-111.

26. MCCLARY Susan, «Getting Down off the Beanstalk: The Presence of a Woman's Voice in Janika Vandervelde’s Genesis II », Minnesota Composers' Forum Newsletter (Fév. 1987), p. 8.

27. MCCLARY Feminine Endings, op. cit., p. 128.

28. CITRON Marcia, "Gender, Professionalism and the Musical Canon ", The Journal of Musicology Vol. 8, No. 1 (hiver 1990), p. 102-117 ; CITRON, Marcia, Gender and the Musical Canon, Cambridge, Cambridge University Press, 1993.

29. FRITH Simon, GOODWIN Andrew (eds.), On Record. Rock, Pop and the Written Word, Londres et New York, Routledge, 1990.

30. LLEDO Eugène, « Rock et séduction », Vibrations, hors-série, Rock : de l'histoire au mythe, MIGNON Patrick et HENNION Antoine (dirs), 1991, p. 121-144.

31. FAST Susan, « Rethinking Issues of Gender and Sexuality in Led Zeppelin: A Woman's View of Pleasure and Power in Hard Rock », American Music 17/3 (Automne 1999), p. 245-299.

32. FAUSER Annegret, "L'élément érotique dans l'œuvre de Massenet ", in Massenet en son temps, Actes du colloque organisé en 1992 à l'occasion du deuxième Festival Massenet, Saint-Etienne, Association du festival Massenet/L'Esplanade Saint-Etienne Opéra, 1999, p. 156-179.

33. KERMAN Joseph, "How We Got into Analysis How to Get Out ", Critical Inquiry Vol. 7, No. 2 (hiver 1980), p. 311-331. [ "Comment nous sommes entrés en analyse, et comment en sortir », in Du politique dans l'analyse musicale, Esteban BUCH, Nicolas DONIN et Laurent FENEYROU eds., Paris, Vrin, « Musicologies », 2013, p. 33-51].

34. Camille Bellaigue, cité en FAUSER, op. cit.

35. DENORA Tia, « Music and Erotic Agency - Sonic Resources and Socio-Sexual Action », Music \& Body 3/2 (1997), 43-65. DENORA, Tia, Music in Everyday Life, Cambridge, Cambridge University Press, 2000.

36. MCQUINN, Julie, "Exploring the Erotic in Debussy's Music", The Cambridge Companion to Debussy, TREZISE Simon (éd.), Cambridge et New York, Cambridge University Press, 2003.

37. FINK Robert, Repeating Ourselves. American Minimal Music as Cultural Practice, Berkeley et Los Angeles, University of California Press, 2005 ; Earogenous Zones: Sound, Sexuality and Cinema, JoHnson Bruce (éd.), London et Oakville, Equinox, 2010.

38. LAZZARO Federico, «Chansons madécasses, modernisme et érotisme. Pour une écoute de Ravel au-delà de l'exotisme », Revue musicale OICRM 3/1 (2014), en ligne.

39. GIOIA Ted, Love Songs: The Hidden History, Oxford, Oxford University Press, 2015.

40. RAYKOFF Ivan, Dreams of Love. Playing the Romantic Pianist, Oxford, Oxford University Press, 2014.

41. PÉRÉA François, Le dire et le jouir. Ce qu'on se dit au lit, Paris, La Musardine, 2017.

42. SOFER Danielle, «Specters of Sex: Tracing the Tools and Techniques of Contemporary Music Analysis ", Zeitschrift der Gesellschaft für Musiktheorie 17/1 (2020), p. 31-63.

43. SAVigliano Marta, Tango and the Political Economy of Passion, Boulder, Westview Press, 1994.

44. Versión. Estudios de comunicación y política No 33 : Música, sexualidad y género, SEMÁN Pablo et SPATARO Carolina (sld), mars 2014, en ligne ; CAROzZI María Julia, Aquí se baila el tango. Una etnografía de las milongas porteñas, Buenos Aires, Siglo XXI, 2015 ; LISKA Mercedes, Entre géneros y sexualidades: Tango, baile, cultura popular, Buenos Aires, Milena Caserola, 2018.

45. MCCLARY Susan, Ouverture féministe. Musique, genre, sexualité, trad. DEUTSCH Catherine et ROTH Stéphane, Paris, La Découverte/Philharmonie de Paris, «La rue musicale », 2015.

46. DEUTSCH Catherine, Maddalena Casulana ou la preuve par l'exemple: musique et philogynie dans l'Italie de la première modernité, mémoire d'HDR, Paris, Sorbonne Université, 2020. 
47. koestenbaum Wayne, Anatomie de la folle lyrique, trad. BURY Laurent, Paris, Philharmonie de Paris/La Découverte, « La rue musicale », 2019.

48. Transposition. Musique et sciences sociales $\mathrm{n}^{\circ} 3$ : Musique et théorie queer, CONTRERAS ZUBILLAGA Igor (sld), 2013, en ligne.

49. ЈАСОтот Sophie, Danser à Paris dans l'entre-deux-guerres: Lieux, pratiques et imaginaires des danses de société des Amériques (1919-1939), Paris, Nouveau Monde, 2013 ; CLAIRE Élizabeth, « Monstrous Choreographies: Waltzing, Madness, and Miscarriage ", Studies in Eighteenth-Century Culture, vol. 38, 2009, p. 199-235 ; CLAIRE Élizabeth (dir.), CLIO. Femmes, genre, histoire $\mathrm{n}^{\circ} 46$ : Danser, numéro coordonné par Florence ROCHEFORT et Michelle ZANCARINI-FOURNEL, 2017.

50. BUCH Esteban, «Climax as Orgasm: On Debussy's L'isle joyeuse », Music and Letters 100/1 (Fév. 2019), p. 24-60.

\section{INDEX}

Mots-clés : Études de genre, New Musicology, Musique et sexualité, L'Inondation, Chloé Briot Keywords : Gender Studies, New Musicology, Music and Sexuality, L'Inondation, Chloé Briot

\section{AUTEURS}

\section{ESTEBAN BUCH}

Né en 1963 à Buenos Aires, Esteban Buch est directeur d'études à l'École des Hautes Études en Sciences Sociales (EHESS) de Paris. Spécialiste des rapports entre musique et politique, il est l'auteur, notamment, de Trauermarsch. Daniel Barenboïm et l'Orchestre de Paris dans l'Argentine de la dictature (Éditions du Seuil, 2016), O juremos con gloria morir. Una historia del Himno Nacional Argentino (Buenos Aires, Eterna Cadencia, 2013), L'affaire Bomarzo. Opéra, perversion et dictature (Éditions de l'EHESS, 2011), Le cas Schönberg. Naissance de l'avant-garde musicale (Gallimard, 2006), La Neuvième de Beethoven. Une histoire politique (Gallimard, 1999) et Histoire d'un secret. À propos de la Suite Lyrique d'Alban Berg (Actes Sud, 1994). Il a coédité plusieurs ouvrages collectifs, dont La Grande Guerre des musiciens (Symétrie, 2009), Du politique en analyse musicale (Vrin, 2013), Composing for the State (Routledge, 2016) et Finding Democracy in Music (Routledge, 2021). Il prépare actuellement l'ouvrage Playlist. Musique et sexualité.

\section{VIOLETA NIGRO GIUNTA}

Violeta Nigro Giunta est pianiste et musicologue. Doctorante à l'EHESS/CRAL sous la direction d'Esteban Buch, elle mène une thèse sur les transformations dans le champ de la musique contemporaine à Buenos Aires après 1983. Elle a présenté ses recherches dans d'événements scientifiques tels que LASA, ARLAC et CISH. Elle a coorganisé les conférences Instrumental Theatre: Music and the Stage in Latin America, 1954-2006 (Buenos Aires, 2018) et Le son et la musique au prisme des sound studies (Paris, 2019). Elle est l'auteure de nombreuses publications dont les dernières sont "Defining Audible Democracy: New Music in Post-Dictatorship Argentina" (Esteban Buch and Robert Adlington (eds.), Finding Democracy in Music, London, Ashgate, 2020) et "The Sound of the 2001 Argentine Crisis" (Michael Bull and Marcel Corbussen (eds.), The 
Bloomsbury Handbook of Sonic Methodologies, London, Bloomsbury, 2020). Elle a donné des cours à l'EHESS et à Sciences Po (Paris) et elle est codirectrice de la revue Transposition. Musique et Sciences Sociales. 